\title{
Ozonetherapy vs SARS-COV-2 in gut: the fountainhead
}

by Giovanni T. Ranaldi, MD, Emanuele Rocco Villani, MD, Laura Franza, MD, Giulia Motola, MD. Symple Clinical Pharmacology \& Clinical Trial Operating Unit, Az. Sanitaria Potenza, Potenza, Italy ranalgio@tiscali.it Department of Geriatrics, Catholic University of the Sacred Heart, Rome, Italy, emanuele.rocco.villani@gmail.com Department of Emergency Medicine, A. Gemelli University Hospital Foundation IRCCS, Rome, Italy, cliodnaghfranza@gmail.com

Primary Cares Complex Operating Unit, Healthcare Authority Potenza, Italy, giulia.motola@aspbasilicata.it

Correspondence to: Emanuele Rocco Villani, MD, ORCID 00000001-88132419,emanuele.rocco.villani@gmail.com

\begin{abstract}
COVID-19 is the disease caused by the new coronavirus SARS-CoV-2 and is characterized by clinical manifestations ranging from mild, flu-like symptoms, to severe respiratory failure and multiorgan failure. Patients with more severe symptoms may require intensive care treatments and face a high risk of mortality.

COVID 19 is characterized by an abnormal inflammatory response similar to a cytokine storm, which is associated with endothelial dysfunction and microvascular complications. To date, no specific treatments are available for COVID-19 and its potentially life-threatening complications.

Experimental and clinical observations have suggested that the gut microbiota plays a key role in sepsis and ARDS pathogenesis. The incidence of diarrhea in COVID-19 patients and the high mortality rate in elderly patients, considered together, indicate a possible involvement of the intestinelung axis in COVID-19 with association of dysbiosis.

Ozonetherapy is the administration of a mixture of ozone and oxygen, or Medical Ozone (MO), which produces a series of benefits capable of counteracting a wide range of pathologies, in use for over a century as an unconventional medicine practice.

MO is fundamental to inhibit the activation of the inflammatory reaction and to obtain an antioxidant activity both in the tissue and in the blood. Oxidative ozone preconditioning causes an increase in SOD, GSH-Px values.

MO, using large auto-hemo-infusion or rectal insufflation technique or ozonized water, could help oxygenate the tissues better, decrease gut inflammation and regulate immune response, help slow down viral growth, especially in the early stages, with obvious benefit at microbiome level.
\end{abstract}

Key-word: ozone; ozonetherapy, SARS-COV-2, COVID-19; endothelial dysfunction; microcirculation; acute respiratory distress syndrome; cytokine storm, small bowel, gut, microbioma, dysbiosis. 


\section{Introduction}

Coronavirus 2019 (COVID-19) disease is caused by the new SARS-CoV-2 virus, first reported in December 2019 in Wuhan, Hubei province, China. Symptoms range from a mild cough to pneumonia or it can run without symptoms. There is evidence that it spreads from person to person and to date has affected more than 22 million people, causing 780,000 deaths worldwide.

One of the serious clinical manifestations of Covid-19 is pneumonia and progression to acute respiratory distress syndrome (ARDS), especially in elderly patients with immune compromise. Experimental and clinical observations have suggested that gut microbiota plays a key role in the pathogenesis of sepsis and ARDS. [1]

Viral RNA was found in approximately $53 \%$ of the patient's stool samples sampled and gastrointestinal manifestations were found in 5-30\% of patients with SARS-CoV-2 lung infection. Respiratory viruses can change intestinal microbiota composition and the influence of these viruses on the intestinal microbiota can be mediated by systemic signals, physiological changes and increased susceptibility to colitis. [2]

COVID-19 patients are characterized by less bacterial diversity, with relative abundance of opportunistic pathogens, such as Streptococcus, Rothia, Veillonella and Actinomyces, and depletion of beneficial populations. H1N1 patients showed lower diversity and a different overall microbial composition than COVID-19 patients. [3]

SARS-CoV-2 infection does not only remain in the respiratory tract and the gastrointestinal system also contributes to the course of the disease. The small bowel likely plays a much larger role in Covid19 dynamics, as it is likely a key site of the origin with systemic inflammatory response syndrome (SIRS) and "cytokine storm" amplification.

SARS-COV-2s could move to the systemic circulation after damage to the lung tissue and migrate to the intestinal cells through the circulatory and lymphatic system. In this way, virus can avoid the harsh environment of the gastrointestinal tract with gastric and intestinal fluid which can disrupt virus lipid envelope and inhibit its infectivity. [4]

\section{The intestine as an organ}

With its 32 square meters of surface, the intestine is the largest internal organ of the human body: evolution has meant that, alongside the basic task of taking on, digesting and absorbing nutrients, the intestine also carried out numerous other fundamental activities, such as immune system organ, as organ with endocrine activity and as "microbiological apparatus", besides being rich in neuronal cells, "the secon brain".

In general, the histological organization is the same from the esophagus to the wall of the large intestine, with a mucous tunic, a submucous tunic, a muscle tunic and a serous tunic.

The intestinal mucosa is composed of several elements to form the physical and immunological defense barrier, i.e. the external mucus layer with intestinal commensal bacteria, antimicrobial peptides (AMP) and secretory IgA molecules, the layer of specialized epithelial cells and the internal lamina propria containing innate and adaptive immune principles such as $\mathrm{T}$ cells, B cells, macrophages and dendritic cells. 
The intestinal epithelium is a single layer of cells that line the intestinal lumen, which not only prevents the passage of harmful intraluminal entities (antigens, microorganisms and their toxins) but also acts as a selective filter to allow the translocation of essential dietary nutrients, electrolytes and water from the intestinal lumen in the circulation

Intestinal permeability is regulated by multiple factors including exogenous factors, epithelial apoptosis, cytokines and immune cells. In addition, the intestinal lumen contains millions of microorganisms. Intestinal epithelial cells (IEC) must tolerate commensal microbiota bacteria presence and therefore must not respond to their products, but still protect the intestinal mucosa from potentially harmful food antigens and invading pathogens.

IEC express a variety of innate immune receptors, such as Toll-like receptors (TLR), to detect endogenous microbes and warning signs. Intestinal intraepithelial lymphocytes (IELs) reside within the IEC and participate in intestinal mucosa barrier formation. In case of pathogens invasion, the deregulation of the immunity of the mucous membrane or the damage to the IEL causes the interruption of intestinal homeostasis and exacerbates inflammation [5].

The human intestinal microbiota is made up of $10^{14}$ resident microorganisms which include bacteria, arcs, viruses and fungi. The host's interactions with the microbiota are complex, numerous and bidirectional. The gut microbiota significantly regulates the development and function of the innate and adaptive immune system. Intestinal diners secrete antimicrobial peptides, compete for nutrients and the habitat site thus helping in maintaining homeostasis. Intestinal microbiota-derived signals tune immune cells for pro and anti-inflammatory responses, thereby influencing susceptibility to various diseases. [6]

Intestinal microbiota alterations sometimes collectively called "intestinal dysbiosis" have been shown to be associated with various diseases and disorders such as inflammatory bowel syndrome (IBD), type 2 diabetes, depression, cardiovascular disease.

Immune bowel homeostasis is orchestrated by regulatory balance fine tuning which is ultimately controlled by commensal microorganisms. Microorganisms serve as a source of molecular schemes associated with microorganisms (MAMP) and molecular schemes associated with pathogens (PAMP). The two are recognizable on host cells through pattern recognition receptors (PRR), which include TLRs and nucleotide binding receptors. TLRs recognize MAMPS and PAMP among other molecules and evoke different immunological reactions depending on the type of cell, ligand or receptor. [7]

As with the gut microbiota, there is evidence to suggest the presence of distinct microorganisms in the lung [8]. Intestinal and lung microbiota have been shown to influence lung health through a vital cross-dialogue between gut and lung which is called "intestine-lung axis". The gut-lung axis should be bidirectional, which means that endotoxins, microbial metabolites can affect the lung through the blood and inflammation in the lung, can also affect the gut microbiota [9]. This raises the possibility that the new SARS-Cov2 may also have an impact on the gut microbiota.

Airway microbiota composition has been extensively studied in patients with chronic airway diseases of both infectious and non-infectious origin, less in patients suffering from acute lung infections, such as pneumonia: however, the beneficial role of the host microbiota during acute bacterial lung infections have been shown in numerous settings using GF mice that are more sensitive to lung infections from Pseudomonas aeruginosa, Streptococcus pneumoniae and Klebsiella pneumoniae. [10] 
ARDS is a common and serious COVID-19 complication and evidence shows that the lung microbiota of patients with ARDS is different from that of patients without ARDS; therefore, changes in the microbial composition in the lung of COVID-19 patients may predict ARDS.

Antibiotic-treated mice have been reported to be more sensitive to respiratory pathogens such as S. pneumoniae and K. pneumoniae. In particular, mice treated with antibiotics and infected with S. pneumoniae show a defect in lung cytokines production. Oral stool transplantation with a normal intestinal microbiota has restored both the infection control in mice and cytokines levels in lungs, showing the contribution that the intestinal microbiota makes to lung immunity. $[9,11]$

Finally, dysbiosis in the intestinal microbiota contributes to disease pathogenesis in respiratory tract. Chronic lung diseases, such as chronic inflammatory lung diseases and asthma, have been shown to occur mainly in presence of gastrointestinal tract diseases such as IBD and in patients showing altered structure and function of intestinal mucosa and impaired permeability.

Interestingly, the fecal metabolomic analysis suggested potential amino acid-related pathways that link the gut microbiota to inflammation, explaining the predisposition of some individuals to develop severe COVID-19. [12]

SARS-CoV-2-associated diarrhea may appear even before respiratory symptoms and some patients showed only digestive symptoms. [13]

\section{SARS-COV-2 and intestine}

Covid-19 begins with SARS-COV-2 binding to the cell and its subsequent penetration: SARS-CoV2 binds to the transmembrane protein ACE2 via the viral Spike (S) protein, triggered by the cell protease TMPRSS2, to enter the Type II alveolar epithelial cells, macrophages, pericytes and other cell types including epithelial and gland cells in the esophagus and enterocytes in the ileum and colon. Indeed, the small bowel has the largest number of ACE2 receptors in the human body.

SARS-CoV-2 has a higher affinity for binding to ACE2 than SARS-CoV and involves a greater number of interaction sites [14] and ACE2 blockage leads to increased angiotensinogen levels and renin-angiotensin system (RAS) hyperactivation, leading to systemic vasoconstriction and SIRS.

ACE2 has a function independent of RAAS: in the intestine it regulates amino acids absorption and intestinal bacterial balance, helping to reduce intestinal inflammation with the expression of the neutral amino acid transporter B0AT1 in intestinal epithelial cells. [15]

In ACE2-B0AT1 complex absence the serum levels of the neutral amino acids valine, threonine, tyrosine and tryptophan amino acid (Trp) are reduced, causing severe intestinal inflammation and microbial imbalance, as demonstrated in mutant Ace2 mice.

Amino acids and nicotinamide can activate mTOR, which is involved in cell proliferation, survival, protein synthesis and transcription. Reduction in mTOR activity has recently been reported in mice lacking B0AT1. mTOR inhibition with rapamycin in wildtype mice resulted in significant downregulation of antimicrobial peptide expression. [15]

Tryptophan regulates the expression of mRNA antimicrobial peptides through the mTOR pathway, and antimicrobial peptides can influence intestinal microbiota composition [16]. Consequently, the downregulation of ACE2 by reducing the intestinal absorption of tryptophan reduces the secretion of antimicrobial peptides with greater survival of the pathogens and intestinal dysbiosis. Pathogenic 
bacteria such as Clostridium hathewayi, Bacteroides nordii and Actinomyces viscosus start to predominate.

No other organ in the body has a larger number of lymphocytes, which also play a fundamental role in mediating SIRS. SARS-CoV-2 induced ACE2 blockage, gut microbiome alterations and bacterial or toxin translocation are major initiators and drivers of SIRS. This can lead to the vasculitis and thrombosis seen in patients with Covid-19 [17]

The high levels of pro-inflammatory cytokines circulating in viral infections are capable of altering the intestinal microbiota and disturbing intestinal integrity. A malfunction in the small intestine leads to an altered intestinal microbiota and inflammation due to the unbalanced bidirectional interaction between the intestinal microbiota and the immune system.

Inflammatory cytokines such as TNF- $\alpha$ induce a calcium dependent increase in mitochondrial reactive oxygen species (ROS). Furthermore, interferon- $\gamma$ has been shown to upregulate genes by inducing the generation of mitochodral ROS. IL-6 and IL-10 have been found to modulate mitochondrial ROS generation by modulating electron transport chain activity and stimulate proinflammatory cytokine production. Several lines of evidence have established a link between inflammation and oxidative stress. [18]

When the mitochondria have been seriously damaged, their content is released into the cytosol and into the extracellular environment, with the production of pro-inflammatory cytokines and activation of the NLRP3 and IL-6 inflammasomes. [19]

NLRP3 has been shown to be closely associated with Crohn's disease and can be triggered, with the essential role of mitochondria, by a variety of stimuli, such as infections and tissue damage, and therefore activated through different cellular signaling pathways. Upon activation, the NLRP3 inflammasome causes the maturation and secretion of pro-inflammatory cytokines IL-1 $\beta$ and IL-18: the activation of Nrf2 inhibits NLRP3 expression, caspase-1 activation and IL-1 $\beta$ secretion. [20,21]

In stressful conditions such as viral infections, mitochondria can modulate immune responses leading to increased inflammation [22]. This unbalanced immune response can cause microbiotic dysbiosis. Furthermore, mitochondria have been shown to alter the microbial community by influencing the activities of intestinal functional effector cells, such as immune cells, epithelial cells and enterochromaffinic cells.

The increase in inflammation in the intestine leads to a loss of the intestine which allows antigens and bacterial toxins to move into the systemic circulation, further worsening the septic state of patients with COVID-19: therefore, a hesitant inflammatory reaction may have started in multiple organ failure. Bacterial translocation from the intestine to the lungs has been reported in sepsis and acute respiratory distress syndrome due to possible barrier dysfunction.

Finally, intestinal injury resulting from ischemia/reperfusion (I/R) is an important clinical event in disorders such as trauma, burns, septic or hypovolemic shock etc. and plays an important role in the pathogenesis of systemic inflammation and multiple organ failure (MOF). Intestinal I/R is also a critical and trigger event in the development of distal organ dysfunction, which frequently involves the lungs. Respiratory failure is a common cause of death and complications after intestinal I/R. [23]

Clinical and experimental studies suggest that oxidative stress induced by reactive oxygen species is one of the most important mediators in the pathogenesis of intestinal I/R and it causes the dysregulated production of molecules such as hydrogen peroxide (H2O2), superoxide and inflammatory cytokines 
which they can damage distant organs. This leads to the development of a systemic inflammatory response syndrome, which can progress to MOF [18]. Intestinal I/R lesions also cause lung neutrophil infiltration, which contributes to the development of acute respiratory distress syndrome. Hence, the intestine was referred to as "the engine of the MOF". [24]

\section{Ozone therapy and intestine}

Gastrointestinal administration of medical ozone (MO) was initially supported by Aubourg who described the intrarectal administration of an ozone/oxygen mixture for the treatment of chronic colitis and fistulas [25]. Over the years, the positive effects of MO therapy in various gastrointestinal disorders have been demonstrated, such as intestinal lesions induced by methotrexate, incompetence of intestinal anastomosis sutures, experimental colitis, preventive effect of ozone in experimental models of necrotizing enterocolitis, burns caustic esophageal and acute necrotizing pancreatitis.

The trophic effect of MO on the intestine is demonstrated by the increase in the weight of the mucosa, mucosa DNA and proteins content, height of the villi and depth crypt compared to control animals. The most significant changes were observed in the proximal intestine.

Parallel increases in DNA and intestinal mucosa proteins indicate that the increased mucosal mass of animals treated with ozone can be attributed to cell hyperplasia and exclude factors such as edema or congestion.

The increase in villi height may be the result of greater proliferation and accelerated migration along the villi, and is an indicator of the greater absorbent surface. The increase in crypt cells together with hypertrophy of individual cells is characteristic of tissues subjected to increased cell proliferation or repair.

MO-treated rats demonstrated a significant increase in cell proliferation compared to control animals and parenteral MO exerted a significantly greater stimulating effect compared to oral administration. [26]

Cell loss in the small intestine is primarily regulated by programmed cell death or apoptosis, a highly regulated and reproducible event that ultimately leads to cell death. Several regulatory genes that influence apoptosis have been identified and divided into pro-apoptotic and anti-apoptotic genes. MO treatment resulted in a significant reduction in enterocytic apoptosis and caspase-3 protein levels. [27]

MO direct effect can be related to the production of $\mathrm{H} 2 \mathrm{O} 2$ : after administration, $\mathrm{MO}$ dissolves in biological fluids, reaches the intestinal mucosa and immediately reacts with polyunsaturated fatty acids, antioxidants, reduced glutathione and albumin, resulting in the formation of $\mathrm{H} 2 \mathrm{O} 2$ and lipid oxidation products (LOPS).

$\mathrm{H} 2 \mathrm{O} 2$ is able to act as $\mathrm{MO}$ messenger to elicit numerous biological and therapeutic effects, including the regulation of signal transduction, and is a local defenses and immune system responses facilitator.

MO indirect effects on intestinal mucosal homeostasis can be related to various beneficial pharmacological effects, such as reduction of edema, impaired adhesion of leukocytes, enhancement of antibacterial activity, stimulation of fibroblastic proliferation, neo -vascularization and the release of the growth factor from platelets or macrophages. Recent evidence suggests that MO has modulatory effects on the phagocytic activity of peritoneal and alveolar macrophages. 
MO has been shown to increase the activity of antioxidant enzymes, such as glutathione peroxidase and catalase, which protect the host from pathological conditions ROS mediated. [27]

As seen previously, I/R usually occurs in critically ill patients and leads to systemic inflammation and MOF and oxidative stress is the main mechanism that causes I/R injury.

MO beneficial effects as antioxidant agent have been shown in various pathologies. MO intervenes on cellular antioxidant systems including glutathione, SOD and enzymatic reactions, preparing the host to face the pathophysiological conditions mediated by oxidative stress with repetitive doses.

In the I/R lesion, oxidative stress is followed by a cascade that involves the activation of inflammatory cells, the production of systemic inflammatory mediators, the increase in bacterial translocation, the release of bacterial products such as endotoxin and leading to their systemic diffusion, SIRS and MOF.

Recently, intrarectal application of MO has been shown to reduce ROS by stimulating and/or preserving endogenous antioxidant systems in experimental reperfusion models of hepatic and renal ischemia. [28]

Preconditioning with MO reduced the pulmonary wet/dry ratio, neutrophil infiltration, lipid membrane peroxidation and increased SOD activity in the lungs after intestinal I/R. Consistent with these observations, histopathological analysis also indicated that preconditioning could improve lung injury induced by intestinal I/R injury. [29]

MO administration at the level of the arteriopathies of the lower limbs and of the disc-radicular conflict blocks the inflammation processes through molecular pathways linked to Nf-kB and Nfr2. $\mathrm{NF}-\kappa \mathrm{B}$ and Nrf2 are part of an important network of transcription factors and regulatory proteins that modulate the expression of a wide range of genes, including those associated with inflammatory responses. The activation of Nf-kB promotes the transcription of genes linked to pro-inflammatory cytokines, while MO exerts the anti-inflammatory and consequently anti-apoptotic capacity, blocking the action of NF- $\kappa \mathrm{B}[30]$ through the upregolation of the $\mathrm{Nfr} 2$ route.

Nrf2 is a main regulator of cellular oxidative levels against environmental stresses by inducing the expression of metabolic detoxification and antioxidant enzymes to eliminate ROS and the gastrointestinal tract is a key source of ROS. The intestinal barrier is essential for maintaining the healthy stable state of the human gastrointestinal tract and Nrf2 has been shown to play an important role in maintaining the integrity of the intestinal mucosa barrier. [31]

Oxidative stress is one of the main engines of the wrong folding of proteins since it induces the oxidation of proteins and Nrf2 promotes the clearance of oxidized or otherwise damaged proteins through the two main pathways of protein degradation, namely the ubiquitin proteasome system and autophagy.

Consistently, MO treatment has been shown to promote wound healing by increasing fibroblast migration through the phosphoinositide 3-kinase (PI3K)/protein kinase B (Akt)/mTOR signaling pathway [32].

The Nrf2-Keap1 pathway participates in many other cellular protection mechanisms as well as counteracting oxidative stress, infacts [33]:

(1) reduced lesion of the intestinal mucosa by suppressing oxidative stress;

(2) decreased intestinal inflammation by inhibiting the inflammatory pathway; 
(3) has affected intestinal tight junction proteins and cell apoptosis to regulate intestinal permeability;

(4) influenced the differentiation and functions of T cells;

(5) crossregulation between the KEAP1-NRF2 pathway and intestinal oxidative stress controlled by autophagy.

Activation of Nrf2 can alter the Th1/Th2 balance as demonstrated by changes in cytokine production: Th1/Th2 differentiation is a critical process for the host's immunological defense in the intestine. Activation of Th1 cells eliminates intracellular pathogens by activating macrophages and releasing various cytokines. Th2 cells stimulate B cells to secrete antibodies and play important roles in defending the host against worm infection. [34].

The abundance of innate and adaptive immune cells resides in close contact with trillions of commensal microorganisms in the human gastrointestinal tract, which requires barrier and regulatory mechanisms that limit host-microbial interactions and maintain tissue homeostasis. Th1/Th2 differentiation is a critical process in adapting adaptive immune responses against commensal bacteria [35].

Activation of Nrf2 by tBHQ significantly reduced IFN- $\gamma$ production markedly in the splenocytes stimulated with $\mathrm{CD} 3 / \mathrm{CD} 28$ in mice. Furthermore, the activation of Nrf2 promoted Th2 differentiation of CD4 + T cells, inhibiting the Th1 differentiation shown by the corresponding changes in Th1/Th2 cytokine production.

Nrf2-/- mice have increased endotoxin plasma levels compared to wild-type mice in intestinal barrier injury. [36]

The increase in cellular Nrf2 causes the transcriptional activation of the gene for mTOR, Nrf2 directly regulates mTOR transcription when the PI3K path is intact, while this function is lost when PI3K is activated. We identified a link between the environmental stress detection system Nrf2 and mTOR, which is a key cellular protein in metabolism. The transcriptional regulation Nrf2 of mTOR is related to the promoter binding of $\mathrm{p} 65$ and to the methylation of the histone residues permitting transcription. $\mathrm{Nrf} 2$ can act upstream of the PI3K path and transcriptionally regulates one of its main components, namely mTOR.

Nrf2 has been shown to indirectly up-regulate mTOR activity by increasing the expression of RagD, a protein known as mTOR activator. The absence of Nrf2 induces more aggressive inflammation through the activation of p65 and proinflammatory cytokines downstream in astrocytes. Furthermore, the activation of antioxidant signaling mediated by $\mathrm{Nrf} 2$ attenuates the inflammatory response mediated by NF- $\kappa \mathrm{B}$ in a carcinoma model of the colorectal associated with colitis and Nrf2 - / - mice showed an increase in lung activity of NF- $\kappa \mathrm{B}$ and an inflammatory response after a traumatic brain injury. [37]

\section{Conclusions}

SARS-CoV-2 infection does not only remain in the respiratory tract and the gastrointestinal system also contributes to the course of the disease. The small bowel likely plays a much larger role in the dynamics of Covid-19, as it is likely a key site of the origin with amplification of the SIRS and the "cytokine storm". 
The use of MO through the techniques of large auto-hemo-infusion, rectal insufflation or the intake of ozonated water, could contribute in addition to better oxygenating the tissues, monitoring lung inflammation and regulating the immune response, slowing growth viral, to produce beneficial effects in terms of safeguarding the intestinal structure, intestinal inflammation and damage to the microbiome. [38].

By bubbling the ozone in the water, water with a certain amount of dissolved ozone is obtained.

The use of ozonated water is widely used for various gastric pathologies such as Elicobacter pilori gastritis, which become negative after about 15 days of therapy and is also indicated to treat those affected by colitis, chronic diseases of the large intestine, duodenitis. [39]. Oral use of ozonated oil has been shown to be effective in the treatment of Giarda Lamblia infestation and intestinal malabsorption syndrome [40]. The use of ozonnated water can also act as a preconditioning of the intestine and microbiota and its regular use increases immune defenses against most diseases.

On the other hand, the action of ozone at the microcirculation level is well known $[41,42]$ and its action depends on the direct or indirect generation of important mediators such as $\mathrm{H} 2 \mathrm{O} 2$, 4 HNE, NO, QNO1, HO1 and the regulation of the Pathways Nrf2-NF-Kb and Angpt1 / Tie 1-2, to counteract the overproduction of early-response proinflammatory cytokines (TNF, IL-6 and IL-1 $\beta$ ) and ultimately to the fulminating multi-organ failure that we have also seen active intestinal.

In this perspective, it becomes important to consider MO in Covid-19 therapy, since there are no major side effects and being able to be synergistic with other therapies, it is a candidate to be essential therapy in home care, given the ease of execution and the low cost.

Finally, in particular cases, the use of the ozone-dilution technique in physiological solution and its infusion could also be evaluated and, in the future, the possibility of using ozone therapy with endonasal insufflation as a substitute for the vaccine could be evaluated [43].

\section{References}

1. Dhar D, Mohanty A. Gut microbiota and Covid-19- possible link and implications. Virus Res. 2020; 285: 198018. doi: 10.1016 / j.virusres.2020.198018.

2. He Y, Wang J, Li F, Shi Y. Main Clinical Features of COVID-19 and Potential Prognostic and Therapeutic Value of the Microbiota in SARS-CoV-2 Infections. Front Microbiol. 2020; 11: 1302. Published 2020 Jun 5. doi: 10.3389 / fmicb.2020.01302.

3. Gu S, Chen Y, Wu Z, et al. Alterations of the Gut Microbiota in Patients with COVID-19 or H1N1 Influenza [published online ahead of print, 2020 Jun 4]. Clin Infect Dis. 2020.

4. Aktas B, Aslim B. Gut-lung axis and dysbiosis in COVID-19. Turk J Biol. 2020; 44 (3): 265 272. Published 2020 Jun 21. doi: 10.3906 / biy-2005-102.

5. Wen Z, Liu W, Li X, et al. A protective role of the NRF2-Keap1 pathway in the maintenance of intestinal barrier function. Oxid Med Cell Longev. 2019; 2019: 1,759,149.

6. Negi S, Das DK, Pahari S, Nadeem S, Agrewala JN. Potential Role of Gut Microbiota in Induction and Regulation of Innate Immune Memory. Front Immunol. 2019; 10: 2441.

7. Dhar D, Mohanty A. Gut microbiota and Covid-19- possible link and implications. Virus Res. 2020; 285: 198018.

8. Bingula, Rea \& Filaire, Marc \& Radosevic-Robin, Nina \& Bey, Mathieu \& Berthon, JeanYves \& Bernalier-Donadille, Annick \& Vasson, Marie-Paule \& Filaire, Edith. (2017). Desired Turbulence? Gut-Lung Axis, Immunity, and Lung Cancer. Journal of Oncology. 2017. 1-15. 
9. Dumas A, Bernard L, Poquet Y, Lugo-Villarino G, Neyrolles O. The role of the lung microbiota and the gut-lung axis in respiratory infectious diseases. Cell Microbiol. 2018; 20 (12): e12966.

10. Fagundes CT, Amaral FA, Vieira AT, et al. Transient TLR activation restores inflammatory response and ability to control pulmonary bacterial infection in germfree mice. $\mathrm{J}$ Immunol. 2012; 188 (3): 1411-1420.

11. Schuijt TJ, Lankelma JM, Scicluna BP, et al. The gut microbiota plays a protective role in the host defense against pneumococcal pneumonia. Gut. 2016; 65 (4): 575-583.

12. Wanglong Gou, View ORCID ProfileYuanqing Fu, Liang Yue, Geng-dong Chen, Xue Cai, Menglei Shuai, Fengzhe Xu, Xiao Yi, Hao Chen, View ORCID ProfileYi Judy Zhu, Mian-li Xiao, Zengliang Jiang, Zelei Miao, Congmei Xiao, View ORCID ProfileBo Shen, Xiaomai Wu, Haihong Zhao, Wenhua Ling, Jun Wang, View ORCID ProfileYu-ming Chen, View ORCID ProfileTiannan Guo, View ORCID ProfileJu-Sheng Zheng, Gut microbiota may underlie the predisposition of healthy individuals to COVID-19, https://doi.org/10.1101/2020.04.22.20076091.

13. Chen N Zhou M Dong X Qu J Gong F Epidemiological and clinical characteristics of 99 cases of 2019 novel coronavirus pneumonia in Wuhan, China: a descriptive study. The Lancet. 2020; 395: 507.

14. Hoffmann M, Kleine-Weber H, Schroeder S, Kruger N, Herrler T, Erichsen S, Schiergens TS, Herrler G, Wu NH, Nitsche A, Muller MA, Drosten C, Pohlmann S. SARS-CoV-2 cell entry depends on ACE2 and TMPRSS 2 and is blocked by a clinically proven protease inhibitor. Cell 2020.

15. Hashimoto, T.; Perlot, T.; Rehman, A. ACE2 links amino acid malnutrition to microbial ecology and intestinal inflammation. Nature 2012, 487, 477-481.

16. Liévin-Le Moal V, Servin AL. The front line of enteric host defense against unwelcome intrusion of harmful microorganisms: mucins, antimicrobial peptides, and microbiota. Clin Microbiol Rev. 2006; 19 (2): 315-337.

17. Mönkemüller K, Fry LC, Rickes S. Systemic inflammatory response and thrombosis due to alterations in the gut microbiota in COVID-19 [published online ahead of print, 2020 Jun 24]. Rev Esp Enferm Dig. 2020; 112: 10.17235 / reed.2020.7297 / 2020. doi: 10.17235 / reed.2020.7297 / 2020.

18. Khomich OA, Kochetkov SN, Bartosch B, Ivanov AV. Redox Biology of Respiratory Viral Infections. Viruses. 2018; 10 (8): 392. Published 2018 Jul 26. doi: 10.3390 / v10080392.

19. Mittal M, Siddiqui MR, Tran K, Reddy SP, Malik AB. Reactive oxygen species in inflammation and tissue injury. Antioxid Redox Signal. 2014; 20 (7): 1126-1167.

20. Xiuting Liu, Wei Zhou, Xin Zhang, Ping Lu, Qianming Du, Lei Tao, Yang Ding, Yajing Wang, Rong $\mathrm{Hu}$, Dimethyl fumarate ameliorates dextran sulfate sodium-induced murine experimental colitis by activating Nrf2 and suppressing NLRP3 inflammasome activation, Biochemical Pharmacology, Volume 112, 2016, Pages 37-49.

21. Yanghao Hou, Yueting Wang, Qi He, Lingyu Li, Hui Xie, Yong Zhao, Jing Zhao, Nrf2 inhibits NLRP3 inflammasome activation through regulating Trx1 / TXNIP complex in cerebral ischemia reperfusion injury, Behavioral Brain Research, Volume 336, 2018, Pages 32- 39.

22. Green DR, Galluzzi L, Kroemer G. Mitochondria and the autophagy-inflammation-cell death axis in organismal aging. Science. 2011; 333 (6046): 1109-1112. 
23. IH Mallick, W. Yang, MC Winslet, and AM Seifalian, "Ischemia-reperfusion injury of the intestine and protective strategies against injury," Digestive Diseases and Sciences, vol. 49, no. 9, pp. 1359-1377, 2004.

24. DN Granger, M. Hollwarth, and DA Parks, "Ischemia-reperfusion injury: role of oxygenderived free radicals," Acta Physiologica Scandinavica, vol. 548, pp. S47 - S63, 1986.

25. Gao C, Sun X, Zhang G, et al. Hyperoxygenated solution preconditioning attenuates lung injury induced by intestinal ischemia reperfusion in rabbits. J Surg Res. 2008; 146 (1): 24-31.

26. Sukhotnik I, Starikov A, Coran AG, Pollak Y, Sohotnik R, Shaoul R. Effect of ozone on intestinal epithelial homeostasis in a rat model. Rambam Maimonides Med J. 2015; 6 (1): e0006. Published 2015 Jan 29. doi: 10.5041 / RMMJ.10181.

27. Haj, B., Sukhotnik, I., Shaoul, R. et al. Effect of ozone on intestinal recovery following intestinal ischemia - reperfusion injury in a rat. Pediatr Surg Int 30, 181-188 (2014).https://doi.org/10.1007/s00383-013-3448-8.

28. Onal O, Yetisir F, Sarer AE, et al. Prophylactic Ozone Administration Reduces Intestinal Mucosa Injury Induced by Intestinal Ischemia-Reperfusion in the Rat. Mediators Inflamm. 2015; 2015: 792016. doi: 10.1155 / 2015/792016.

29. Changjun Gao, Xude Sun, Guihe Zhang, Hui Zhang, Hui Zhao, Yonghui Yang, Lichun Han, Lixian Xu, Wei Chai, Hyperoxygenated Solution Preconditioning Attenuates Lung Injury Induced by Intestinal Ischemia Reperfusion in Rabbits, Journal of Surgical Research, Volume 146, Issue 1, 2008, Pages 24-31, ISSN 0022-4804, https://doi.org/10.1016/j.jss.2007.07.008.

30. Re L, Martínez-Sánchez G, Bordicchia M, Malcangi G, Pocognoli A, Morales-Segura MA, et al. Is the ozone pre-conditioning effect related to the Nrf2 / EpRE activation pathway in vivo? A preliminary result. European Pharmacology Review. 2014; 742: 158-62.

31. Galiè M, Covi V, Tabaracci G, Malatesta M. The Role of Nrf2 in the Antioxidant Cellular Response to Medical Ozone Exposure. Int J Mol Sci. 2019; 20 (16): 4009. Published 2019 Aug 17. doi: 10.3390 / ijms20164009.

32. Xiao W., Tang H., Wu M., Liao Y., Li K., Li L., Xu X. Ozone oil promotes wound healing by increasing the migration of fibroblasts via PI3K / Akt / mTOR signaling pathway. Biosci. Rep. 2017; 37 doi: 10.1042 / BSR20170658.

33. Wen Z, Liu W, Li X, et al. A Protective Role of the NRF2-Keap1 Pathway in Maintaining Intestinal Barrier Function. Oxid Med Cell Longev. 2019; 2019: 1,759,149. Published 2019 Jun 26. doi: 10.1155 / 2019/1759149.

34. Zagorski JW, Turley AE, Dover HE, VanDenBerg KR, Compton JR, Rockwell CE The Nrf2 activator, tBHQ, differentially affects early events following stimulation of Jurkat cells. Toxicological Sciences. 2013; 136 (1): 63-71.

35. Peterson LW, Artis D. Intestinal epithelial cells: regulators of barrier function and immune homeostasis. Nature reviews Immunology. 2014; 14 (3): 141-153.

36. Wen Z, Liu W, Li X, et al. A Protective Role of the NRF2-Keap1 Pathway in Maintaining Intestinal Barrier Function. Oxid Med Cell Longev. 2019; 2019: 1,759,149. Published 2019 Jun 26. doi: 10.1155 / 2019/1759149.

37. Bendavit G, Aboulkassim T, Hilmi K, Shah S, Batist G. Nrf2 Transcription Factor Can Directly Regulate mTOR: linking cytoprotective gene expression to a major metabolic regulator that generates redox activity. J Biol Chem. 2016; 291 (49): 25476-25488.

38. D'Ambrosio CM: Treatment of chronic inflammatory bowel diseases by rectal oxygenozone therapy. Italian Journal of Oxygen-Ozone Therapy 1: 155-158, 2002.

39. Souza Yglesio Moyses de, Fontes Belchor, Martins Joilson O, Sannomiya Paulina, Brito Glacus S., Younes Riad N. et al. Evaluation of the effects of ozone therapy in the treatment 
of intra-abdominal infection in rats. Clinics [Internet]. 2010 [cited 2020 July 14]; 65 (2): 195-202.

40. Roosevelt Luis Cambara Pena, Roosevelt Cambara Valdes, et al., Ozone therapy, LibrosEn Red, 2017

41. Ranaldi GT, Villani ER, Franza L. Rationale for ozone-therapy as an adjuvant therapy in COVID-19. Med Gas Res [Epub ahead of print] [cited 2020 Jul 13].

42. Ranaldi, GT, MD, Villani, ER, Franza, L., \& Motola, G. (2020, June 3). Devils and Angels: Ozonetherapy for microcirculation in covid-19. https://doi.org/10.31226/osf.io/c2jvt.

43. Ranaldi, Giovanni T., Emanuele R. Villani, and Laura Franza. 2020. "Ozonetherapy: A Multirole Weapon, The Topical Pathway against SARS-COV-2": https://doi.org/10.31226/osf.io/n2dup. 